\section{Exzessiver fragmentarischer Myoklonus}

Helga Peter

Marburg, Deutschland

\section{Synonyme}

EFM

\section{Englischer Begriff}

excessive fragmentary myoclonus

\section{Definition}

Exzessiver fragmentarischer Myoklonus findet sich als elektromyographischer Zufallsbefund in der > ,Polysomnographie“"während des NREM-Schlafs. Es handelt sich um kleine zuckende Bewegungen der Mundwinkel, Finger oder Zehen, sichtbare Bewegungen können aber auch fehlen. Die Betrof- fenen sind sich der Myoklonien meist nicht bewusst. Assoziationen mit fast allen schlafmedizinischen Erkrankungen sowie der Niemann-Pick-Erkrankung Typ C bei Kindern wurden beschrieben. Die Myoklonien können aber auch isoliert auftreten. Bisher ist unklar, ob die Myoklonien Ursache oder Folge von Schlafunterbrechung sind. Es gibt keine Anhaltspunkte für auslösende Faktoren oder einen rückwirkenden Einfluss auf die bestehende jeweilige Grunderkrankung. Der Verlauf der Störung ist kaum untersucht; sie scheint benigne zu sein und zeigt keine Progression. Für einen Zusammenhang mit neurodegenerativen Erkrankungen gibt es bisher keinen Anhaltpunkt.

Der Nachweis von EFM erfolgt mittels > ,Polysomnographie“, am besten mit mehreren EMG-Ableitungen. Die Auswertung erfolgt nach den Kriterien des $>$ „AASM-Manual“. EFM muss bei der Differentialdiagnostik anderer Schlafbezogener Bewegungsstörungen (siehe $>$,Schlafbezogene Bewegungsstörungen“) berücksichtigt werden.

Die Internationale Klassifikation der Schlafstörungen von 2014 ( „,ICSD-3“) zählt den Exzessiven fragmentarischen Myoklonus zu den Isolierten Symptomen und Normvarianten unter den Schlafbezogenen Bewegungsstörungen. 